Relations industrielles

Industrial Relations

\title{
Borrel, Monique, Conflits du travail, changement social et politique en France depuis 1950
}

\section{Jacques-André Lequin}

Volume 52, numéro 3, 1997

URI : https://id.erudit.org/iderudit/051196ar

DOI : https://doi.org/10.7202/051196ar

Aller au sommaire du numéro

Éditeur(s)

Département des relations industrielles de l'Université Laval

ISSN

0034-379X (imprimé)

1703-8138 (numérique)

Découvrir la revue

Citer ce compte rendu

Lequin, J.-A. (1997). Compte rendu de [Borrel, Monique, Conflits du travail, changement social et politique en France depuis 1950]. Relations industrielles / Industrial Relations, 52(3), 664-667. https://doi.org/10.7202/051196ar

Tous droits réservés @ Département des relations industrielles de l'Université Laval, 1997
Ce document est protégé par la loi sur le droit d'auteur. L’utilisation des services d’Érudit (y compris la reproduction) est assujettie à sa politique d'utilisation que vous pouvez consulter en ligne.

https://apropos.erudit.org/fr/usagers/politique-dutilisation/ 
individuelle se replie pour mieux résister à l'isolement du travailleur et à l'omnipotence de l'employeur, que se passe-til lorsque l'action collective cesse d'unifier et commence à diviser les travailleurs en gagnants et perdants, lorsque le travailleur ne doit plus etre protégé contre l'employeur mais contre la collectivité à laquelle il appartient " (p. 229) [ ?]

Il y va de la justification, dans l'un et l'autre droit, de la pratique de plus en plus contemporaine de la négociation dite "de gestion", des accords de solidarité, défensifs ou offensifs, favorables à l'emploi, mais emportant, par exemple des diminutions de rémunération pour des salariés en Italie, ou encore, en France, des accords dérogatoires ou, plus généralement, du développement des accords " donnant-donnant ». Certes, l'autonomie collective ne pourra disposer que des droits des travailleurs qui ne sont pas " indisponibles par nature", comme le seraient par exemple ceux leur résultant d'une loi impérative. L'analyse conduit ici à envisager la notion d'avantages individuels acquis. Mais, une seconde condition s'ajoute: la légitimité des organisations syndicales appelées à s'engager ainsi dans une telle négociation de gestion. Leur représentativité légitime-t-elle l'édiction de normes contraignantes emportant renonciation à des droits individuels des salariés? Une réponse négative parait s'imposer, puisque le « concept de représentativité se dissocie de l'idée de représentation ", pose-t-on (p. 341), après analyse (pages 300 et suivantes). Le recours au procédé référendaire apporterait un correctif approprié, de suggérer l'auteure.

Le caractère "donnant-donnant " est un caractère typique de la négociation collective nord-américaine, s'il était à nouveau permis d'extrapoler. Mais, alors représentativité et représentation légale se confondent et ont précisément pour fondement la volonté majoritaire au sein de la collectivité des salariés en cause.

L'évocation, si incomplète, du contenu de l'ouvrage, "dont la richesse ne nuit jamais à la rigueur de la construction ", comme le dit avec on ne peut plus de justesse le professeur Antoine Lyon-Caen dans sa préface, aura voulu signaler la grande portée de l'analyse des institutions essentielles des rapports collectifs du travail à laquelle il nous convie.

PIERRE VERGE

Université Laval

\section{Conflits du travail, changement social et politique en France depuis 1950}

par Monique BORREL, Paris: L'Harmattan, 1996, 266 p., ISBN 2-7384-4429-5.

À la façon de Shorter et Tilly (Strike in France: 1830-1968, Cambridge: Cambridge University Press, 1974) et plus récemment de Franzosi (The Puzzle of Strike: Class and State Strategies in Postwar Italy, Cambridge: Cambridge University Press, 1995), l'auteure veut comprendre les conflits du travail à travers une vision historique de l'évolution de la situation sociopolitique française. Cette recension porte sur son traitement de la notion de conflits du travail puisque c'est là son sujet principal. Une recension privilégiant une approche plus sociologique serait aussi profitable pour aborder les chapitres traitant de la notion de conflit, de politiques sociales ou de mobilisation syndicale. La présentation de cette recension se calque sur la démarche de l'auteure. Le livre se divise en trois parties.

La première partie "La reconstitution des séries statistiques de conflits du travail " introduit d'abord une contribution originale de l'auteure qui classifie les grèves selon leur ampleur en trois grandes catégories : (1) Les conflits localisés, c'est-à-dire les luttes de nature économique, limitées dans le temps et l'espace (l'entreprise ou l'établissement) visant à 
obtenir une amélioration ponctuelle de leur situation matérielle. (2) Les conflits généralisés et les vagues de grèves ou luttes de nature sociétale, soit un mouvement de grève affectant simultanément un grand nombre d'entreprises (ou d'établissements d'une même entreprise) sur des revendications communes. Leur finalité est de modifier radicalement la nature même du salariat afin de conquérir un statut qui garantisse une amélioration régulière et institutionnalisée des conditions matérielles de vie et de travail. (3) Les journées nationales d'action, soit les luttes de nature politique. Leur objectif a été d'aider la gauche à conquérir le pouvoir (p. 4-5).

Ensuite, cette première partie a un aspect très technique dans le sens où l'auteure explique les problèmes méthodologiques qu'implique la reconstitution de séries statistiques à partir de différentes sources plus ou moins compatibles entre elles (p. 10-13). L'activité de grève n'est mesurée que par le nombre de journées individuelles perdues. Notons que l'auteure ne classifie pas les conflits selon cette dernière mesure, mais selon les caractéristiques de chaque conflit. Ainsi, pour la période de 1950 à 1987, elle énumère et décrit brièvement près de 1500 conflits généralisés et 500 journées nationales d'action (p. 14-20 et 2443). Il est cependant dommage que l'auteure ne nous présente pas les mesures élémentaires de l'activité de grève: la fréquence, la taille et la durée. En effet, cette mesure globale peut cacher des formes d'activité de grève fort différentes.

La deuxième partie "Un modèle économétrique des grèves, de la politique sociale, de l'audience syndicale et de l'électorat en France " constitue l'assise théorique de l'ouvrage et la présentation des résultats des analyses statistiques. Elle se subdivise en sept chapitres.

1. Fondements théoriques de l'analyse du changement social et politique. Ici, l'auteure développe trois thèmes: la place du conflit, la critique de l'hypo- thèse de la rationalité à laquelle elle préfère celle du homo sociologicus intentionnel et les théories de l'action collective où elle retient surtout celle de la mobilisation des ressources. En discutant la place du conflit, elle suggère une théorie globalisante et originale des grèves sur trois axes. L'axe l de la croissance se situe dans l'économie de marché, le conflit est celui du salariat us patronat sur les conditions de travail. L'axe 2 a trait à la redistribution de la croissance qui oppose la gauche à la droite politique quant à l'importance souhaitable du rôle de l'État et de ses politiques socioéconomiques. L'axe 3 concerne les modes de régulation propres à gérer les tensions sur les deux premiers axes (p. 47-52).

\section{Théories et modèles économétriques} des grèves. L'auteure consacre les deux premières sections à discuter des théories et de l'étude de l'activité de grève. Dans les quatre sections suivantes, elle s'adresse au cas français. Après avoir fait la synthèse et critique des théories " classiques " du conflit industriel (théorie du cycle économique, théorie de l'institutionnalisation et le modèle politique), elle ajoute un élément moins connu: la théorie des vagues de grèves (p. 65-71). Cette théorie se comprend mieux si on accepte que la grève peut être à la fois la variable dépendante et indépendante. Les quatre dernières sections seront traitées globalement puisque les thèmes développés seront repris dans les chapitres suivants. Les résultats à venir annoncent que l'explication de l'activité de grève en France dépend du type de grève. Les conflits localisés - lutte économique - répondent bien aux modèles des cycles économiques. Les conflits généralisés et les vagues de grèves - lutte sociétale répondent à des besoins de changements sociaux. Les journées nationales d'action - lutte politique - supposent une coordination des groupes et partis de gauche pour remporter le pouvoir politique. Les résultats statistiques vont dans ce sens (p. 79-87, voir aussi la troisième partie). 
3. Problèmes méthodologiques. Ces problèmes sont de trois types. (1) Les sources d'information, c'est-à-dire comment pallier l'absence de statistiques officielles valides et les problèmes liés à l'utilisation des journaux? (2) Comment tenir compte de la spécificité des pays et comment découper les périodes historiques? (3) Comment quantifier les phénomènes politiques et sociaux? (p. 89-108).

4. Les conflits localisés et les conflits généralisés: principaux déterminants. Comme prévu, les conflits localisés répondent bien aux modèles économiques (p. 109-114). Cependant ils ne sont pas imperméables aux variables qui influencent d'avantage les conflits généralisés. Ces derniers, par leur nature sociétale, supposent une plus grande mobilisation et répondent aux disparités sociales. Cependant, ces deux types de conflits ne sont pas indépendants des luttes politiques. Ainsi lorsque les partis de gauche négocient intensément afin d'avoir une stratégie commune à la veille d'une échéance électorale importante "les travailleurs [ne sont] plus psychologiquement disponibles pour s'engager dans des conflits " (italique dans le texte, p. 111).

Afin de comprendre les conflits de travail en tant que forme de lutte politique, l'auteure consacre les deux chapitres suivants à développer une théorie au sujet de l'État et de la mobilisation des travailleurs au sein du mouvement syndical. De ces chapitres elle construira, entre autres, des variables indépendantes pour expliquer les journées nationales d'action.

5. La politique sociale: principaux déterminants. Dans une première section, l'auteure critique les théories "classiques" de l'État-providence. Puis elle présente succinctement une cinquantaine d'études portant sur l'une ou l'autre de ces théories (p. 125-145). Finalement, elle propose un modèle de la politique sociale à travers les changements socioéconomiques et politiques qui ont marqué la
France, changements qu'elle mesure par l'évolution des «trois composantes stratégiques de la politique sociale française: les prestations sociales, le SMIC et la durée de travail ». Elle retient aussi les fluctuations des rapports droite/gauche dans la vie politique française (p. 145154).

6. L'audience syndicale: principaux déterminants. Bien que l'audience syndicale soit un concept différent et plus large du taux de syndicalisation, il n'existe pas de façon fiable de la mesurer. Elle revoit donc les principaux modèles expliquant le taux de syndicalisation puis se concentre sur les années d'après-1968 en France. Comme indice de syndicalisation, elle utilise le nombre de délégués syndicaux selon la loi et le nombre d'adhérents aux diverses organisations syndicales (p. 166-173).

7. Les journées nationales d'action et la stratégie de conquête du pouvoir politique par la gauche. "L'ampleur des vagues de grèves et des conflits généralisés de 1950 à 1968 a incité les partis politiques de gauche à coordonner leurs stratégies [...] pour orchestrer des journées nationales d'action " (p. 175). Cette coordination se mesure par les accords entre les mouvements de gauches (Parti communiste et Parti socialiste) accords électoraux et de programme, CGT et CFDT. Les analyses statistiques de l'auteure confirment le lien entre la coordination de la gauche et les journées nationales d'action. De plus, de 1967 à 1981 on constate des liens positifs entre la coordination de la gauche, le nombre de journées nationales d'action et l'augmentation des suffrages à la gauche aux différents paliers de gouvernements (p. 177-181).

La troisième partie "Résultats des régressions et tableaux statistiques " présente les résultats des analyses de régression multiple discutées dans la deuxième partie et les tableaux qui permettent d'établir les différentes variables sociopolitiques. Ces présentations statistiques sont 
accompagnées de notes qui, entre autres, rappellent les événements historiques pertinents pour aider à comprendre la réalité que veulent décrire ses résultats statistiques (p. 185-229).

En conclusion, l'auteure résume l'évolution de l'activité de grève. Au lendemain de la Deuxième Guerre, les travailleurs utilisent la grève pour améliorer leurs conditions économiques et réduire les écarts entre eux et la bourgeoisie. En France, cette activité de grève se généralise et provoque des vagues de grève pour forcer l'État à jouer un rôle plus actif dans la protection des travailleurs. Bref, que les gains locaux chez les grands employeurs, dans les secteurs publics et nationalisés soient partagés par l'ensemble de la classe ouvrière. Ainsi émerge l'État-providence. Les partis de gauche, réalisant la force de l'action de grève, s'en servent comme outil de mobilisation - journées d'action nationale - pour conquérir le pouvoir politique local, régional et national (p. 233-236).

L'auteure a bien réussi sa tâche. Évidemment, ce livre est très important pour ceux qui s'intéressent à l'activité de grève en France. Il faut souligner l'effort gigantesque qu'il a fallu pour reconstituer des séries statistiques cohérentes de l'activité de grève depuis 1950. L'auteure a dû poser des jugements et faire des choix lors de cet exercice qui paraissent fort raisonnables et cohérents. Sans être un expert de la situation en France, les séries reconstruites semblent bien rendre compte de ce qui a déjà été publié sur l'expérience française. De plus, les résultats statistiques et leur interprétation ne reposent pas uniquement sur ces choix et jugements. Finalement, l'auteure a inclus dans son ouvrage toutes les informations nécessaires pour bien apprécier ses résultats et pour les reproduire.
Sa théorie de l'activité de grève est particulièrement intéressante. Elle s'articule autour de trois axes et permet de mieux comprendre la nature différente des grèves selon leur ampleur. Cette catégorisation de l'activité de grève pourrait être raffinée. Ainsi, pourquoi avoir regroupé en une seule catégorie les conflits généralisés et les vagues de grèves? Il nous semble que les vagues de grève constituent une catégorie particulière, qu'elles sont comme un signal que des conflits généralisés sont dans une phase de transformation où l'aspect sociétal du conflit cède le pas à la dimension politique. Néanmoins, l'importance et l'originalité de la contribution de l'auteure tiennent davantage dans son approche générale que dans sa catégorisation précise de l'activité de grève.

Ainsi, cet ouvrage présente un intérêt pour l'ensemble des gens qui s'intéressent aux conflits du travail. Dans un contexte nord-américain, cette approche devrait nous permettre de constater que si la majorité des grèves sont des conflits locaux, elles ne le sont pas toutes. Ainsi, au Québec, les vagues de grèves du début des années soixante-dix auraient avantage à être revues selon l'approche proposée par l'auteure. Par ailleurs, il faudrait considérer d'autres types de manifestation qui ne sont pas des grèves au sens du Code du travail (marches des femmes, manifestations pour l'emploi, actions de solidarité ouvrière et populaire). Ces actions collectives se rapprochent de la définition des journées d'action nationale en France.

Bref, la contribution de madame Borrel est importante non seulement pour ses résultats, mais surtout parce qu'elle offre une nouvelle approche pour comprendre et interpréter l'activité de grève.

JACQUES-ANDRÉ LEQUIN Université du Québec à Hull 